\title{
A machine learning approach for detecting CNAME cloaking-based tracking on the Web
}

\author{
Ha Dao \\ The Graduate University for Advanced Studies (Sokendai) \\ Email: hadao@nii.ac.jp
}

\author{
Kensuke Fukuda \\ NII/Sokendai \\ Email: kensuke@nii.ac.jp
}

\begin{abstract}
Various in-browser privacy protection techniques have been designed to protect end-users from third-party tracking. In an arms race against these counter-measures, the tracking providers developed a new technique called CNAME cloaking based tracking to avoid issues with browsers that block thirdparty cookies and requests. To detect this tracking technique, browser extensions require on-demand DNS lookup APIs. This feature is however only supported by the Firefox browser.

In this paper, we propose a supervised machine learning-based method to detect CNAME cloaking-based tracking without the on-demand DNS lookup. Our goal is to detect both sites and requests linked to CNAME cloaking-related tracking. We crawl a list of target sites and store all HTTP/HTTPS requests with their attributes. Then we label all instances automatically by looking up CNAME record of subdomain, and applying wildcard matching based on well-known tracking filter lists. After extracting features, we build a supervised classification model to distinguish site and request related to CNAME cloaking-based tracking. Our evaluation shows that the proposed approach outperforms wellknown tracking filter lists: F1 scores of 0.790 for sites and $\mathbf{0 . 8 8 5}$ for requests. By analyzing the feature permutation importance, we demonstrate that the number of scripts and the proportion of XMLHttpRequests are discriminative for detecting sites, and the length of URL request is helpful in detecting requests. Finally, we analyze concept drift by using the $\mathbf{2 0 1 8}$ dataset to train a model and obtain a reasonable performance on the 2020 dataset for detecting both sites and requests using CNAME cloaking-based tracking.
\end{abstract}

\section{INTRODUCTION}

There are several reasons websites intend to track user's browsing activities. In some acceptable cases, it is simply to make the user browsing experience faster and more convenient. On the other hand, user's browsing activities are also used to determine user browsing habits and preferences for advertisement and analytics on the web, which can be frightful for privacy-sensitive users [1].

Aiming to capture the economic value of user browsing habit through tracking, many entities design services to collect user's information, even without any user's knowledge or consent [2] [3] [4]. There are some effective approaches against third-party tracking for user's privacy-preserving. Ad-blocking and third-party tracking protections have been successfully preventing such trackers using blacklist [5] [6] [7], machine learning-based detection [8] [9], and others [10] [11] [12].

However, third-party web tracking has been getting more sophisticated in an arms race against these counter-measures. One of the current trends is the exploitation of Canonical
Name Record or Alias (CNAME) record in DNS, to bypass the filter lists in browsers and extensions. This technique, called CNAME cloaking-based tracking, uses CNAME to disguise requests to a third-party tracker as first-party ones. The current counter-measures mainly focus on third-party tracking and do not impact first-party resources, CNAME cloaking-based tracking can thus bypass this blocking.

There are some existing methods to detect CNAME cloaking-based tracking. EasyPrivacy [13], AdGuard tracking protection [14], and other filter lists manually add new first party subdomains which are fronts for CNAME cloaking to these blacklists. However, this approach will dramatically increase the size of the blacklists and these subdomains need to be updated frequently. Besides that, uBlock Origin since version 1.24.1b0 performs a DNS lookup of the hostname loading a resource to determine if the underlying subdomain is related to CNAME cloaking or not. Nevertheless, only Firefox allows uBlock Origin to block CNAME cloaking because the other browsers do not support DNS resolution API [7].

In this paper, we propose a machine learning approach to detect CNAME cloaking-based tracking on the web without dynamic DNS lookup. Our method consists of three steps. (1) We crawl a list of target sites and store all HTTP requests with their attributes. Then we look up CNAME record of subdomains, and label all instances by applying wildcard matching based on well-known tracking filter lists on this CNAME records (§ III-B). (2) Next, we extract their features (§ III-C). (3) Finally, we build a supervised classification model to distinguish sites and requests using CNAME cloaking-based tracking (§ III-D).

The contributions of this study are summarized as follows:

- We propose an effective method to detect CNAME cloaking-based tracking. We obtain a F1 score of 0.790 for detecting CNAME cloaking sites and of 0.885 for requests ( $(\mathrm{IV}-\mathrm{A})$.

- We introduce site-related and request-related features for CNAME cloaking detection ( $\S$ III-C). We also make a detailed analysis about the discriminative power of features regarding CNAME cloaking-based tracking detection ( $§$ IV-B).

- We show that our supervised model also works against newly crawled data by analyzing concept drift (§ IV-C). 


\section{BACKGROUND AND RELATED WORK}

\section{A. Background}

1) Browser-based privacy protection techniques: Several browser-based privacy protections have been designed to protect end-users from third-party tracking, such as Ghostery [5], Disconnect [6], and uBlock Origin [7]. They work effectively to detect third-party tracking.

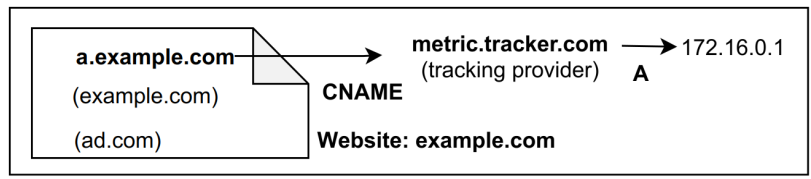

Fig. 1. Overview of CNAME cloaking-based tracking.

2) CNAME cloaking-based tracking: The usage of DNS CNAME records coupled with Content Delivery Network is increasingly commonplace to improve site load times, reduce bandwidth costs, and increase content availability and redundancy. CNAME has also been used for user tracking. Tracking providers ask their clients to delegate a subdomain for data collection and tracking, and link it to an external server using a CNAME DNS record [15]. It behaves differently from ordinary third-party tracking because it uses the firstparty subdomains; browsers and extensions generally do not impact these resources.

For example, when end-users access a site example.com, this site embeds a first-party tracker a.example.com, which points to a tracking provider tracker.com via the CNAME metric.tracker.com. Tracking provider tracker.com thus track activities of end-users on the site example.com (Figure 1).

\section{B. Term definitions}

1) Request linked to CNAME cloaking-related tracking: An HTTP request by a subdomain is used for collecting, and sharing information about a particular user's activities on the web by using CNAME cloaking-based tracking.

2) Site with CNAME cloaking-related tracking occurring: A site sets up a subdomain that points to a tracking provider domain using a CNAME record.

\section{Related work}

1) Anti third-party tracking techniques: The privacy hazards of online web tracking have been studied extensively.

Machine learning-based tracking detection: Harass et al. [9] develop an unsupervised detection method that inspects URL queries in $\mathrm{HTTP}(\mathrm{S})$ requests to detect tracking activities. Yamada et al. [16] analyze traffic at the network gateway to monitor all tracking sites in the administrative network and constructs a graph between sites and their visited time to detect tracking sites. Wu et al. [8] develop DMTrackerDetector which automatically detects third-party trackers offline to efficiently generate blacklists using structural hole theory and supervised machine learning.

Non-machine learning-based tracking detection: Schelter and Kunegis [17] perform a large-scale analysis of third-party trackers by extracting third-party embeddings from more than 41 million domains to study global online tracking. Roesner et al. [11] develop a client-side method for detecting and classifying five kinds of third-party trackers on the 500 most popular and 500 less popular sites according to the Alexa ranking. To cut off the tracking chain of third-party web tracking, Pan et al. [12] develop TrackingFree which isolates unique identifiers into different browser principals so that the identifiers still exist but are not unique among different websites.

2) Existing in-browser CNAME cloaking-based tracking detection techniques: In order to block first-party trackers using CNAME records, uBlock Origin resolves the hostname of a DNS record to determine if an underlying subdomain is related to CNAME cloaking or not. However, only Firefox with DNS API allows uBlock Origin to block the CNAME cloaking [7]. Besides that, the well-know tracking filter lists make an continuous effort to manually update first-party subdomains which are fronts for CNAME cloaking to these blacklists. It makes day-to-day filter lists updating tedious and timeconsuming.

\section{DESIGN AND IMPLEMENTATION}

Here, we describe our method to detect CNAME cloakingbased tracking.

\section{A. Method overview}

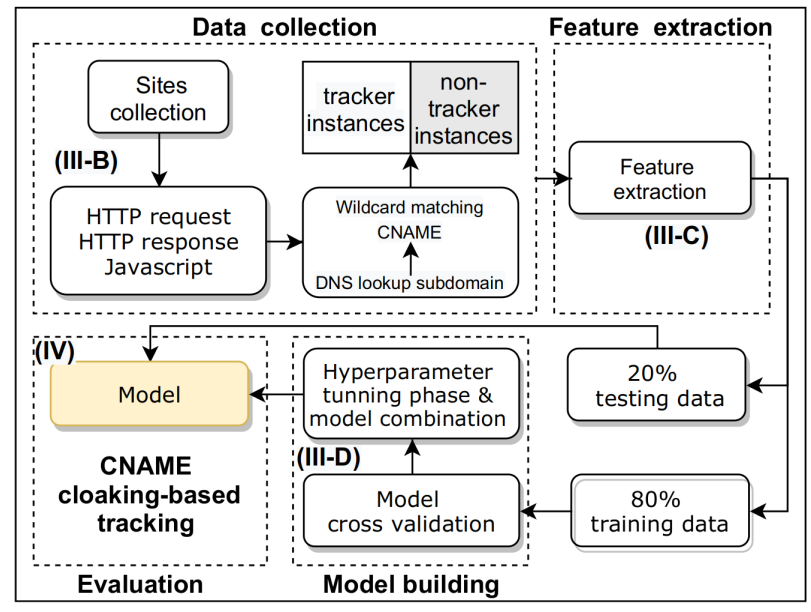

Fig. 2. Processing flow.

Our goal is to develop a CNAME cloaking-based tracking detection method using machine learning. Our method flags sites and requests related to CNAME cloaking-based tracking.

As shown in Figure 2, the method consists of four steps: data collection, feature extraction, model development, and evaluation.

1) Crawl the target sites, including sites with and without CNAME cloaking based tracking, and save all the HTTP requests and their attributes. Then we perform DNS lookup for all subdomains, and label all instances automatically by matching well-known tracking filter lists with CNAME behind all subdomain as described in [18]. 
These instances are divided into two sets, which we call the tracker set and the non-tracker set ( $\S$ III-B).

2) Extract features for sites and requests ( $\S$ III-C).

3) Check the F1 score of 10 classification algorithms using 10-fold stratified cross-validation. After evaluating performance, we select some effective classification algorithms for hyper-parameter tuning phase, and combine conceptually different machine learning classification algorithms to build a model ( $\S$ III-D).

4) Evaluate the models with the testing data ( $\S$ IV).

\section{B. Data collection}

1) Finding CNAME cloaking-based tracking with blacklists: We present our method to label CNAME cloaking-based tracking. Firstly, we perform DNS queries for CNAME answer set on all subdomains of each site. Then, we apply wildcard matching based on well-known tracking filter lists to each resolved CNAME in order to detect CNAME cloaking base tracking. More specifically, we select third-party tracking domains and international third-party tracking domains from Easy privacy list [19] and tracking servers list from AdGuard tracking protection filter [14] as of April 26, 2020. This approach was used in the previous work [18].

2) Datasets: We use OpenWPM [20] with vanilla Firefox in April 2020 to crawl 1,762 sites from Alexa Top 300k where CNAME cloaking-based tracking was previously detected [18]. We also crawl 1,762 sites that were randomly picked from all sites without CNAME cloaking-based tracking [18]. The stored data is all HTTP/HTTPS requests and their responses for each site. Then, applying the method ( $\S$ III-B1) to the stored crawled data, we find that 4,047 HTTP requests in 1,532 sites used CNAME cloaking-based tracking ${ }^{1}$.

We also rely on another dataset crawled on Alexa Top 100K sites using OpenWPM [20] in April 2018. We check the historical forward DNS (FDNS) datasets provided by Rapid7 [21], and apply the methodology in $\S$ III-B1 to detect CNAME cloaking-based tracking in this historical data. We find that 2,546 HTTP requests in 1,005 sites are related to CNAME cloaking-based tracking. We also use 1,005 additional randomly picked sites without CNAME-cloaking from this dataset to analyze concept drift in $\S$ IV-C. The details of the 2020 dataset and the 2018 dataset are listed in Table I.

TABLE I

SUMMARY OF DATA: 2,010 SITES IN 2018 AND 3,524 SITES IN 2020.

\begin{tabular}{llrr}
\hline Metrics & & 2018 & 2020 \\
\hline 3rd party requests & & $185,896(62.2 \%)$ & $276,686(63.8 \%)$ \\
1st party requests & domain & $60,278(20.2 \%)$ & $120,689(27.8 \%)$ \\
& subdomain & $52,476(17.6 \%)$ & $36,399(8.4 \%)$ \\
\hline Total requests & & $298,649(100 \%)$ & $433,774(100 \%)$ \\
\hline Total sites & & 2,010 & 3,524 \\
\hline
\end{tabular}

\section{Feature extraction}

We experimentally extract the following features related to sites and requests.

\footnotetext{
${ }^{1}$ The dataset is available at https://github.com/fukuda-lab/cname-cloaking
}

1) Site with CNAME cloaking-related tracking occurring:

- num_url: The total number of HTTP requests in a site.

- num_1st: The total number of first-party requests in a site.

- num_3rd: The total number of third-party requests in a site.

- pct_script_call: The proportion of fingerprinting-related method calls in a site.

- pct_xhr: The proportion of XMLHttpRequest in a site, which is a built-in browser object that can be used to make HTTP requests in JavaScript to exchange data between the web browser and the server.

- pct_3rd_window: The proportion of requests originating from a third-party DOM window in the window hierarchy [22].

- ranking: The site ranking according to the Alexa ranking [23].

- country: The site country based on Free IP Geolocation API [24].

- category: The site category according to the FortiGuard Web Filtering [25] dataset in April 2020.

2) Request linked to CNAME cloaking-related tracking:

- method: The desired action to be performed for given request.

- is_xhr: The request uses an API that provides scripted client functionality for transferring data between a client and a server.

- content_type: The HTML tag that resulted in a request, such as image, javascript or document, which are defined in this IDL [22].

- len_url: The length of request URL.

- len_sub: The subdomain length of request.

- len_prefix_sub: The subdomain prefix length of request.

- num_prefix_sub: The number of subdomain prefixes.

- prefix_sub_blacklist: The subdomain prefix is among subdomain prefixes in tracking filter lists [14] [19].

- is_sub_dic: The prefix of subdomain is a word in English dictionary.

- entropy_url: The randomness of request URL by calculating the metric entropy from request URL.

- entropy_sub: The randomness of subdomain by calculating the metric entropy from subdomain.

- entropy_prefix_sub: The randomness of subdomain prefix by calculating the metric entropy from subdomain prefix.

\section{Modeling and preliminary results}

First of all, we split the 2020 dataset (Table I) into testing data and training data. The percentage of the data held over for testing is $20 \%$. It is used in $\S$ IV-A to evaluate our model. Next, we describe how to build a classification model to detect CNAME cloaking-based tracking using testing data $(80 \%$ of the 2020 dataset).

1) Model cross-validation: We randomly split the training dataset into 10 smaller sets (folds) without replacement, where nine folds are used for the model training and the remaining 
one fold is for validating. After obtaining 10 performance estimates by repeating this procedure ten times, we take their average as the final performance estimate. We then compare 10 classification algorithms and evaluate their F1 score using this stratified cross-validation procedure on the training data $(80 \%$ the dataset 2020). We employ 10 supervised classification algorithms with scikit-learn module (random_state $=2$ with default parameters):

- Support Vector Classification (SVC)

- Decision Tree

- AdaBoost

- Random Forest

- Extra Trees

- Gradient Boosting

- Multi-layer Perceptron (MLP)

- K-Nearest Neighbors (KNN)

- Logistic Regression

- Linear Discriminant Analysis (LDA)

We use the F1 score for evaluating the performance of the classifiers. Larger values of the F1 score $(\approx 1.0)$ indicate better performance, and lower values $(\approx 0)$ correspond to worse performance. Figure 3(a) shows the F1 scores for the 10 selected algorithms using 10 -fold stratified cross-validation in the 2020 dataset for detecting sites with CNAME cloakingrelated tracking occurring. All classification algorithms have a different detection performance. The most effective classification algorithms are Random Forest, Extra Trees, and Gradient Boosting, while Support Vector Classification, Multilayer Perceptron, and K-nearest Neighbor achieve moderate performances for this dataset.

Figure 3(b) shows the F1 scores for detecting requests linked to CNAME cloaking-related tracking. The most effective classification algorithms are Random Forest and Extra Trees, while SVC shows the worst performance.

2) Hyper-parameter tuning for best models: From the previous performance evaluations, we select RandomForest, ExtraTrees, and GradientBoosting classifiers for the hyperparameter tuning phase for site classification detection. For requests, we select Random Forest and Extra Trees classifiers.

We perform a grid search optimization for all of them regarding F1 score. The parameter setting that gave the best results on the training data are shown in Table II.

3) Model combination: Now, we have a set of closely wellperforming models for detecting site and requests linked to CNAME cloaking-based tracking. In order to balance out their individual weaknesses, we combine conceptually different machine learning classification algorithms and and use the sum of predicted probabilities to predict the class labels.

We thus use VotingClassifier with soft voting to combine the predictions coming from the following supervised classification algorithms: RandomForest, ExtraTrees, and GradientBoosting for detecting sites, and RandomForest and ExtraTrees for detecting requests. The performance of separate algorithms and the combined model are discussed in the next section.
TABLE II

BEST PARAMETER SETTINGS IN TRAINING PHASE REGARDING F1 SCORE.

\begin{tabular}{llrr}
\hline Algorithm & Parameter & Site & Request \\
\hline Random & max_features & 1 & 1 \\
Forest & min_samples_split & 8 & 2 \\
& min_samples_leaf & 3 & 1 \\
& n_estimators & 100 & 300 \\
\hline Extra & max_features & 10 & 1 \\
Trees & min_samples_split & 2 & 2 \\
& min_samples_leaf & 3 & 1 \\
& n_estimators & 300 & 100 \\
\hline Gradient & max_features & 0.3 & Not selected \\
Boosting & min_samples_leaf & 100 & \\
& n_estimators & 300 & \\
& learning_rate & 0.2 & \\
& max_depth & 8 & \\
\hline
\end{tabular}

\section{ClassifiCATION PERFORMANCE ANALYSIS}

We evaluate our model and feature importance regarding detection of CNAME cloaking-based tracking. We also investigate concept drift using our datasets from 2018 to 2020.

\section{A. General results}

1) The performance of model: The results obtained using the test set for sites and requests linked to CNAME cloakingbased tracking detection on $20 \%$ of the 2020 dataset (preserved for this test) are shown in Table III and Table IV. We first show that the F1 score of all classification algorithms improve slightly after the hyper-parameter tuning for both sites and request. Next, the combined model achieves 0.790 of F1 score for detecting sites and 0.885 of $\mathrm{F} 1$ score for detecting requests. These results show that the combination model exhibit similar performance to other classifiers regarding detection of sites linked to CNAME cloaking based-tracking, but that combined model works better for detecting requests. In order to ease following result comparison, we use the combined models for further analyses.

Checking false negatives, we find that some sites/requests linked to CNAME cloaking have the same attributes with sites/requests without CNAME cloaking based tracking. For example, the healthpartners.com linked to tracking provider Adobe, with only six scripts and the proportion XMLHttpRequest in this site is 0.07. For request, the request https://gjr5.yoigo.com/ea.js points to tracking provider Eulerian; its prefix gjr5 is not in the blacklists and it does not contain any user identification. However, we believe that if we crawl more data with the request linked to track provider Eulerian, we can classify this request as CNAME cloakingbased tracking.

TABLE III

RESULTS OBTAINED USING THE TEST SET FOR SITE LINKED TO CNAME CLOAKING-BASED TRACKING DETECTION.

\begin{tabular}{lrrrr}
\hline Score & $\begin{array}{r}\text { RandomForest } \\
\text { Default/Tuned }\end{array}$ & $\begin{array}{r}\text { ExtraTree } \\
\text { Default/Tuned }\end{array}$ & $\begin{array}{r}\text { GradientBoosting } \\
\text { Default/Tuned }\end{array}$ & Combined \\
\hline Precision & $0.777 / 0.784$ & $0.771 / 0.763$ & $0.781 / 0.801$ & 0.794 \\
Recall & $0.730 / 0.748$ & $0.736 / 0.770$ & $0.779 / 0.782$ & 0.785 \\
F1 score & $0.753 / 0.766$ & $0.753 / 0.766$ & $0.780 / 0.791$ & 0.790 \\
\hline
\end{tabular}




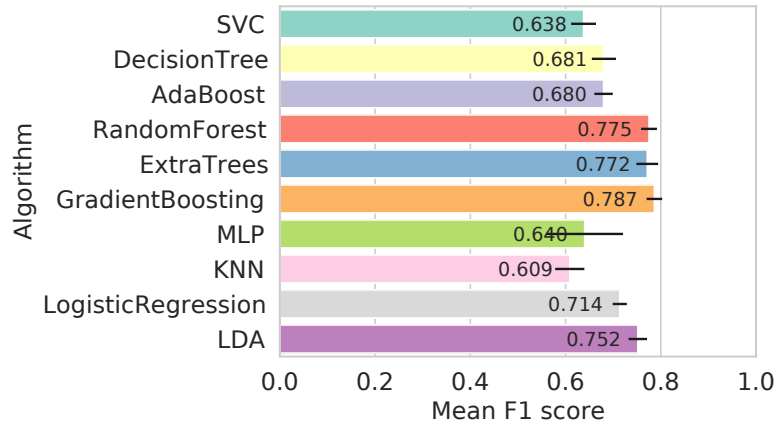

(a) Site linked to CNAME cloaking-based tracking.

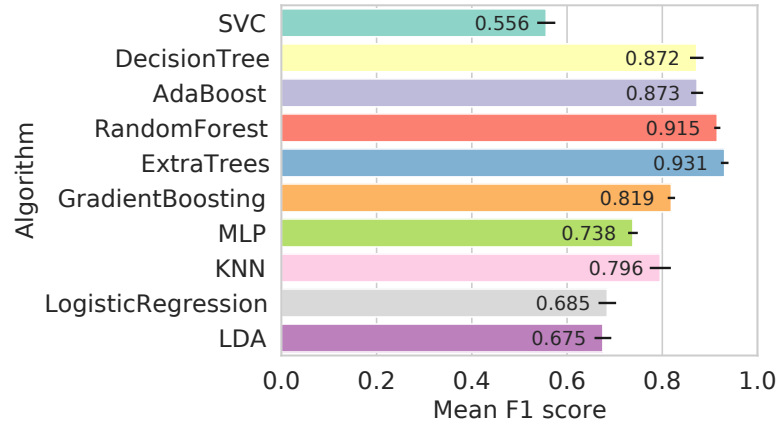

(b) Request linked to CNAME cloaking-based tracking.

Fig. 3. The F1 score for the 10 selected classification algorithms using 10-fold stratified cross-validation in 2020 dataset regarding the detection of CNAME cloaking-based tracking. The mean and standard deviation are computed on the 10 folds of the cross-validation.

TABLE IV

RESULTS OBTAINED USING THE TEST SET FOR REQUEST LINKED TO CNAME CLOAKING-BASED TRACKING DETECTION.

\begin{tabular}{lrrr}
\hline Score & $\begin{array}{r}\text { Random Forest } \\
\text { Default/Tuned }\end{array}$ & $\begin{array}{r}\text { ExtraTree } \\
\text { Default/Tuned }\end{array}$ & Combined \\
\hline Precision & $0.940 / 0.935$ & $0.928 / 0.949$ & 0.949 \\
Recall & $0.807 / 0.830$ & $0.811 / 0.802$ & 0.828 \\
F1 score & $0.869 / 0.879$ & $0.866 / 0.870$ & 0.885 \\
\hline
\end{tabular}

2) Comparison with the well-known tracking filter lists: To block CNAME cloaking-based tracking without DNS resolution, well-known tracking filter lists such as the EasyPrivacy list and the AdGuard tracking filter list include the first-party subdomains which are fronts for CNAME cloaking. We compare the request detection performance between our machine learning approach and these tracking filter lists in Table V. We confirm that our method outperforms the evaluated blocking lists. Meanwhile, almost all browsers (except Firefox) do not provide a DNS resolution API which is critical CNAME cloaking-based tracking detection [18]. Beyond automating blacklist building, our approach is thus especially helpful to block CNAME cloaking-based tracking.

TABLE V

A COMPARISON OF REQUEST DETECTION PERFORMANCE.

\begin{tabular}{lrrr}
\hline Method & Precision & Recall & F1 score \\
\hline Machine learning & $\mathbf{0 . 9 4 9}$ & $\mathbf{0 . 8 2 8}$ & $\mathbf{0 . 8 8 5}$ \\
Easy Privacy list & 0.880 & 0.721 & 0.792 \\
Adguard tracking filter & 0.918 & 0.417 & 0.574 \\
\hline
\end{tabular}

\section{B. Feature permutation importance}

We investigate the permutation importance [26] to discuss the feature importance of the selected classifier.

Figure 4(a) represents the feature permutation importance regarding site detection. We observe that the number of script (num_script) and the proportion of XMLHttpRequest requests (pct_xhr) are the most useful features. In addition, tracking providers are spread across different types of sites [18], site category (category) and site country (country) are thus not helpful in detecting sites with CNAME cloaking-related tracking occurring.
Figure 4(b) shows the feature permutation importance of the model for detecting the requests. The result reveals that the length of request URL (len_url) has the highest importance. We assume that almost requests with a subdomain used for CNAME cloaking-based tracking have a bigger length than the request used for collecting content of site, because they contain user identification. Also, the subdomain prefix length (len_prefix_sub), the XHR usage request (is_xhr), and the randomness of URL request (entropy_url) are discriminative features for request detection. In addition to that, the subdomain prefix blacklist presence (prefix_sub_blacklist) is not effective to detect requests. This is because some publishers also use the same subdomain prefix that is used for both CNAME cloaking and other non-tracking resources.

\section{Concept drift analysis}

Finally, we investigate the ability to detect sites and requests related to CNAME cloaking-based tracking in the latest dataset (2020) using a model trained on the old dataset (2018). We use the 2018 dataset to train a model and test it on new data collected in 2020 (Table I). We apply the method explained in $\S$ III-D to build a model and obtain the results in Table VI.

TABLE VI

RESULTS OBTAINED USING THE NEW SITE/REQUEST IN 2020 DATASET TO EVALUATE 2018 DATASET MODEL.

\begin{tabular}{lrrr}
\hline Instance type & Precision & Recall & F1 score \\
\hline Site & 0.608 & 0.787 & 0.686 \\
Request & 0.935 & 0.558 & 0.699 \\
\hline
\end{tabular}

Our result shows that the performance degradation is limited. Specifically, F1 score for sites with CNAME cloakingrelated tracking decreases by 0.104 , and by 0.186 for requests.

To explain this degradation, we examine the 2018 and 2020 datasets. In 2018, we do not see many random subdomain prefixes and short requests, while it is the case in 2020. For sites, we observe a decreasing number of requests, including third and first-party request for each site in the 2020 dataset. These changes can be plausible reasons for the degradation in our model. Besides that, with rapid changes of web technology, tracking providers might also adjust their target site and change 


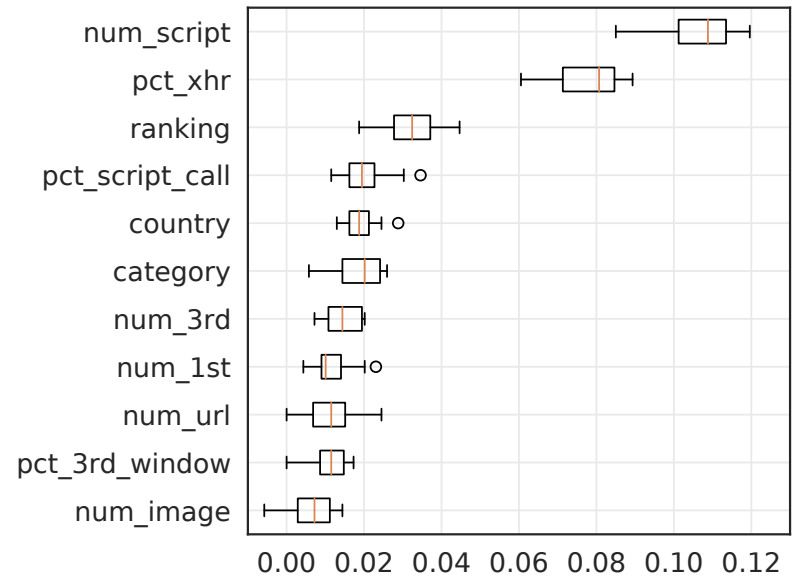

(a) Site linked to CNAME cloaking-based tracking.

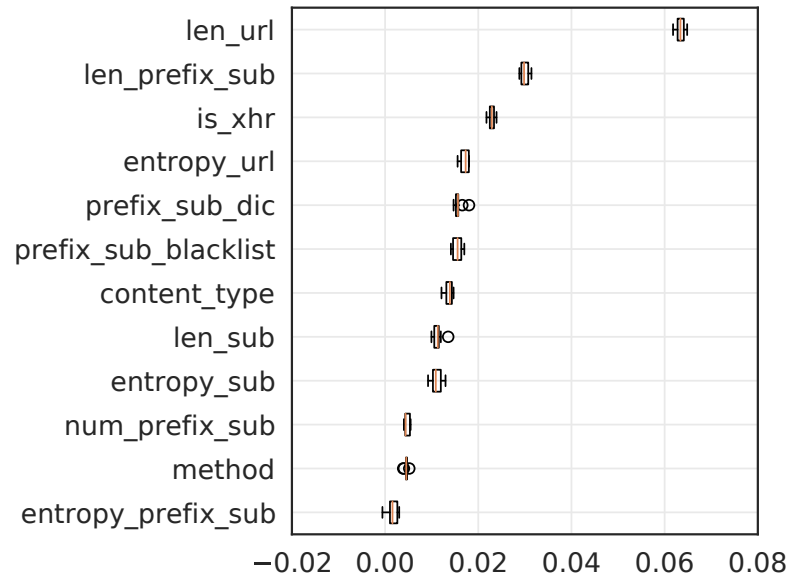

(b) Request linked to CNAME cloaking-based tracking.

Fig. 4. Permutation importance of the combined model for CNAME cloaking-related tracking occurring. The box extends from the lower to upper quartile values of the data, with a line at the median. The number of times a feature is randomly shuffled is $n \_$repeats $=10$.

the implementation methods to deploy CNAME cloakingbased tracking. Although the performance degradation is limited between 2018 and 2020, periodic model retraining can alleviate this problem if more detection accuracy is required.

\section{CONCLUSION AND DISCUSSION}

Recently, CNAME cloaking-based tracking on the web has attracted much attention. The current best countermeasure, blacklist approach, strongly dependant on realtime name resolution. In this paper, we proposed a machine learning approach to detect sites and HTTP requests containing CNAME cloaking-based tracking. Through the comprehensive analysis, we demonstrated the effectiveness of our method.

The proposed method complements existing techniques for CNAME cloaking-based tracking detection and it can help researchers, browser extensions developers, and blacklist maintainers to build highly effective systems against this tracking technique. Once tracking providers know the existence of the proposed detection methodology, they could attempt to evade it. In future work, we intend to add new features to improve our detection performance.

\section{ACKNOWLEDGEMENT}

We thank Johan Mazel for his valuable comments.

\section{REFERENCES}

[1] J. R. Mayer and J. C. Mitchell, "Third-party web tracking: Policy and technology," in IEEE S\&P'12, 2012, pp. 413-427.

[2] G. Acar, C. Eubank, S. Englehardt, M. Juarez, A. Narayanan, and C. Diaz, "The web never forgets: Persistent tracking mechanisms in the wild," in ACM CCS'14, 2014, pp. 674-689.

[3] T.-F. Yen, Y. Xie, F. Yu, R. P. Yu, and M. Abadi, "Host fingerprinting and tracking on the web: Privacy and security implications." in NDSS'12, 2012, pp. 1-16.

[4] H. Metwalley, S. Traverso, M. Mellia, S. Miskovic, and M. Baldi, "The online tracking horde: a view from passive measurements," in IEEE/IFIP TMA'15, 2015, pp. 111-125.

[5] (2009) Ghostery makes the web cleaner, faster and safer! [Online]. Available: https://www.ghostery.com/

[6] (2009) Disconnect. [Online]. Available: https://disconnect.me/
[7] R. Hill. (2014) ublock origin - an efficient blocker for chromium and firefox. fast and lean. [Online]. Available: https://github.com/gorhill/ uBlock

[8] Q. Wu, Q. Liu, Y. Zhang, and G. Wen, "Trackerdetector: A system to detect third-party trackers through machine learning," Computer Networks, vol. 91, pp. 164-173, 2015.

[9] H. Metwalley, S. Traverso, and M. Mellia, "Unsupervised detection of web trackers," in IEEE Globecom'15, 2015, pp. 1-6.

[10] N. Kushmerick, "Learning to remove internet advertisements," in Proceedings of the third annual conference on Autonomous Agents, 1999, pp. $175-181$.

[11] F. Roesner, T. Kohno, and D. Wetherall, "Detecting and defending against third-party tracking on the web," in USENIX NSDI'12, 2012, pp. 1-12.

[12] X. Pan, Y. Cao, and Y. Chen, "I do not know what you visited last summer protecting users from third-party web tracking with trackingfree browser," in NDSS'15, 2015, pp. 1-16.

[13] (2014) Privacy badger - electronic frontier foundation. [Online]. Available: https://www.eff.org/privacybadger

[14] (2014) Adguard tracking protection filter. [Online]. Available: https:// filters. adtidy.org/extension/chromium/filters/3.txt

[15] (2019) Data collection cnames and cross-domain tracking. [Online]. Available: https://docs.adobe.com/content/help/en/id-service/ using/reference/analytics-reference/cname.html

[16] A. Yamada, M. Hara, and Y. Miyake, "Web tracking site detection based on temporal link analysis," in IEEE AINA'10 Workshop, 2010, pp. 626631.

[17] S. Schelter and J. Kunegis, "Tracking the trackers: A large-scale analysis of embedded web trackers," in AAAI ICWSM'16, 2016, pp. 679-682.

[18] H. Dao, J. Mazel, and K. Fukuda, "Characterizing cname cloaking-based tracking on the web," in IEEE/IFIP TMA'20, 2020, pp. 1-9.

[19] (2006) Easyprivacy. [Online]. Available: https://easylist.to/easylist/ easyprivacy.txt

[20] S. Englehardt and A. Narayanan, "Online tracking: A 1-million-site measurement and analysis," in ACM CCS'16, 2016, pp. 1388-1401.

[21] Rapid7 open data, forward dns (fdns). [Online]. Available: https:// opendata.rapid7.com/sonar.fdns_v2/

[22] base - mozsearch. [Online]. Available: https://searchfox.org/ mozilla-central/source/dom/base/

[23] (1996) The top 500 sites on the web. [Online]. Available: https://www. alexa.com/topsites

[24] (unknow) Free ip geolocation api. [Online]. Available: https://freegeoip. app/

[25] (2005) Fortiguard web filtering. [Online]. Available: https://fortiguard. com/webfilter

[26] L. Breiman, "Random forests," Machine learning, pp. 5-32, 2001. 